\title{
Generalized Virtually Stable Maps and Their Associated Sequences
}

\author{
P. Chaoha, ${ }^{1,2}$ S. Iampiboonvatana, ${ }^{1}$ and J. Intrakul ${ }^{1}$ \\ ${ }^{1}$ Department of Mathematics and Computer Science, Faculty of Science, Chulalongkorn University, Bangkok 10330, Thailand \\ ${ }^{2}$ Centre of Excellence in Mathematics, CHE, Si Ayutthaya Road, Bangkok 10400, Thailand \\ Correspondence should be addressed to P. Chaoha; phichet.c@chula.ac.th
}

Received 18 April 2013; Accepted 8 June 2013

Academic Editor: Janusz Matkowski

Copyright (c) 2013 P. Chaoha et al. This is an open access article distributed under the Creative Commons Attribution License, which permits unrestricted use, distribution, and reproduction in any medium, provided the original work is properly cited.

We extend the concept of virtual stability of continuous self-maps to arbitrary selfmaps and investigate the structure of sequences associated with uniformly virtually stable selfmaps. We also obtain a necessary and sufficient condition for a uniformly virtually stable selfmap to have the largest possible associated sequence. Examples of a uniformly virtually stable selfmap having the prescribed largest sequence and a uniformly virtually stable selfmap having no largest sequence are given.

\section{Introduction}

Virtual stability of a self-map was first introduced in [1], where it was proved to unify various (continuous) types of nonexpansiveness in fixed-point theory. The main feature of a virtually stable self-map is that its fixed-point set is always a retract of its convergence set, and this fact immediately allows us to connect topological structures (e.g., connectedness and contractibility) of the fixed-point set to those of the convergence set. Although the original definition of virtual stability requires the continuity of a self-map, it seems that the connection between the fixed-point set and the convergence set also remains valid to some degree in discontinuous settings as hinted in [2, Corollary 2.6] and the work on mean-type mappings in [3-5] (see Example 4 below). A careful investigation of this fact will definitely result in a more powerful notion of virtual stability that enables us to study fixed-point sets of interesting discontinuous self-maps (e.g., Suzuki generalized nonexpansive maps [6]). Moreover, since all well-known self-maps in metric fixed-point theory are always uniformly virtually stable with respect to the same sequence $(n)$ of all natural numbers, it is quite natural to ask whether this also holds in general for a uniformly virtually stable self-map. It turns out that the answer to this simple question is highly nontrivial and interestingly involves algebraic properties of a certain object. Therefore, the purpose of this work is to revise the concept of virtual stability as well as to take care of the previos question. We will first redefine the notion of virtual stability to include discontinuous self-maps and investigate some properties of these newly defined virtually stable self-maps and prove the expected connection between the convergence set and the fixed-point set. Then we will show that the sequence associated with a uniformly virtually stable self-map can be made, in some sense, largest possible provided that the set of all associated sequences has a certain structure. We will also see that when the largest sequence exists, it may not be $(n)$ unless the map is continuous. For the sake of completeness, since the existence of the largest possible sequence associated with a given self-map cannot be guaranteed in general, we will provide two constructive examples of uniformly virtually stable self-maps: one having the prescribed largest sequence and the other having no largest sequence.

\section{Virtual Stability without Continuity}

Let $X$ be a (nonempty) Hausdorff space and $f: X \rightarrow X$ a self-map (that may not be continuous). We will also let $\mathcal{N}(x)$ and $\mathcal{N}_{Y}(x)$ denote the sets of (open) neighborhoods of $x$ in $X$ and in $Y \subseteq X$, respectively. Recall that the fixed-point set and the convergence set of $f$ are defined, respectively, to be $F(f)=\{x: f(x)=x\}$ and

$$
C(f)=\left\{x: \text { the sequence }\left(f^{n}(x)\right) \text { converges }\right\},
$$


where $f^{n}$ denotes the $n$-th iterate of $f$. We also define the map $f^{\infty}: C(f) \rightarrow X$ by

$$
f^{\infty}(x)=\lim _{n \rightarrow \infty} f^{n}(x),
$$

for all $x \in C(f)$. Notice that $f^{\infty}$ may not be continuous, and since we do not assume the continuity of $f$, we only have $F(f) \subseteq f^{\infty}(C(f))$. However, in this work, we will always assume that

$$
\emptyset \neq F(f)=f^{\infty}(C(f))
$$

This condition is quite natural in the sense that it is automatically satisfied whenever $f$ is continuous with $F(f) \neq \emptyset$, and it is also required for the iterative sequence $\left(f^{n}\right)$ to be a scheme according to [2, Definition 2.1]. With the above assumption in mind, we are able to previous define the concept of virtual stability just as in [1, Definition 2.1].

Definition 1. A fixed point $x$ of $f$ is said to be virtually $f$ stable if for each $U \in \mathcal{N}(x)$, there exist $V \in \mathcal{N}(x)$ together with an increasing sequence $\left(a_{n}\right)$ of positive integers such that $f^{a_{n}}(V) \subseteq U$ for all $n \in \mathbb{N}$. We simply call $f$ virtually stable if every fixed point of $f$ is virtually stable. Moreover, we will call a fixed point $x$ of $f$ uniformly virtually $f$-stable with respect to an increasing sequence $\left(a_{n}\right)$ of positive integers if for each $U \in \mathcal{N}(x)$, there exists $V \in \mathcal{N}(x)$ such that $f^{a_{n}}(V) \subseteq U$ for all $n \in \mathbb{N}$. When every fixed point of $f$ is uniformly virtually $f$ stable with respect to the same sequence, we will simply call funiformly virtually stable.

Clearly, a uniformly virtually stable self-map is always virtually stable, and a continuously (uniformly) virtually stable self-map as defined in [1] is also (uniformly) virtually stable according to our new definition. Moreover, if $f$ is uniformly virtually stable with respect to $\left(a_{n}\right)$, it is immediately uniformly virtually stable with respect to any subsequence of $\left(a_{n}\right)$.

Let us recall that, when $(X, d)$ is a metric space, a self-map $f: X \rightarrow X$ is called

(1) virtually nonexpansive [7] if $f$ is continuous and the family $\left\{f^{n}\right\}$ of all iterates of $f$ is equicontinuous on $F(f)$ (equivalently, on $C(f)$ ),

(2) uniformly quasi-Lipschitzian if there is $L>0$ such that

$$
d\left(f^{n}(x), p\right) \leq \operatorname{Ld}(x, p)
$$

for all $(x, p, n) \in X \times F(f) \times \mathbb{N}$.

Notice that a virtually nonexpansive self-map is always continuous and uniformly virtually stable with respect to the sequence $(n)$ of all natural numbers, while a uniformly quasilipschitzian self-map may not be continuous in general.

Proposition 2. Suppose $f$ is a self-map on a metric space $(X, d)$.

(1) If $f$ is continuous and uniformly virtually stable with respect to $(n)$, then it is virtually nonexpansive.
(2) If $f$ is uniformly quasi-lipschitzian, then it is uniformly virtually stable with respect to $(n)$.

Proof. Suppose $f$ is a self-map on a metric space $(X, d)$.

(1) Let $p \in F(f)$ and $\epsilon>0$. Then there is $H \in \mathcal{N}(p)$ such that $f^{n}(H) \subseteq B(p ; \epsilon)$ for all $n \in \mathbb{N}$. Thus, for $\delta>0$ whose $B(p ; \delta) \subseteq H$, we have $f^{n}(B(p ; \delta)) \subseteq$ $f^{n}(H) \subseteq B(p ; \epsilon)$ for all $n \in \mathbb{N}$. This implies that $\left\{f^{n}\right\}$ is equicontinuous on $F(f)$ and hence $f$ is virtually nonexpansive.

(2) Suppose there is $L>0$ such that $d\left(f^{n}(x), p\right) \leq$ $L d(x, p)$ for all $(x, p, n) \in X \times F(f) \times \mathbb{N}$. It follows that $f^{n}(B(p ; r / L)) \subseteq B(p ; r)$ for all $p \in F(f), n \in \mathbb{N}$ and $r>0$. Thus, $f$ is uniformly virtually stable with respect to $(n)$.

It is not difficult to see that the class of uniformly quasilipschitzian self-maps includes various important (possibly discontinuous) self-maps in metric fixed-point theory such as nonexpansive maps, Kannan maps [8], Suzuki generalized nonexpansive maps [6], quasi-nonexpansive maps, and even asymptotically quasi-nonexpansive maps. Hence, the previous proposition immediately implies that those maps are all uniformly virtually stable with respect to $(n)$. The following theorem directly extends [1, Theorem 2.6] to include discontinuous maps.

Theorem 3. Suppose that $X$ is a regular space. If $f$ is virtually stable and $f^{k}$ is continuous for some $k \in \mathbb{N}$, then $f^{\infty}$ is continuous and hence $F(f)$ is a retract of $C(f)$.

Proof. Let $x \in C(f)$ and $U \in \mathcal{N}_{F(f)}\left(f^{\infty}(x)\right)$. Since $X$ is regular, so is $F(f)$ and then there is $W \in \mathscr{N}\left(f^{\infty}(x)\right)$ such that $W \cap F(f) \subseteq \bar{W} \cap F(f) \subseteq U$. By virtual stability of $f, f^{a_{n}}(V) \subseteq W$ for all $n \in \mathbb{N}$, for some sequence $\left(a_{n}\right)$ and $V \in \mathcal{N}\left(f^{\infty}(x)\right)$. Also there is, by the fact that $V \in \mathcal{N}\left(f^{\infty}(x)\right)$, $n_{0} \in \mathbb{N}$ such that $f^{n}(x) \in V$ for all $n \geq n_{0}$. Then $A=$ $f^{-k n_{0}}(V) \cap C(f) \in \mathcal{N}_{C(f)}(x)$ and for each $a \in A$,

$$
\begin{aligned}
f^{\infty}(a) & =\lim _{n \rightarrow \infty} f^{n}(a) \\
& =\lim _{n \rightarrow \infty} f^{a_{n}}\left(f^{k n_{0}}(a)\right) \in \bar{W} \cap F(f) \subseteq U .
\end{aligned}
$$

Thus, $f^{\infty}$ is continuous and hence $F(f)$ is a retract of $C(f)$.

Example 4. Let $p \in \mathbb{N}$ and $p \geq 2$, and let $I \subseteq \mathbb{R}$ be an interval. Consider the subspace $I^{p}$ of $\mathbb{R}^{p}$ equipped with the maximum norm $\left\|\left(x_{1}, \ldots, x_{p}\right)\right\|=\max \left\{\left|x_{1}\right|, \ldots,\left|x_{p}\right|\right\}$. Since the maximum norm on $\mathbb{R}^{p}$ also induces the usual topology, $I^{p}$ is a regular space. Recall that a self-map $f: I^{p} \rightarrow I^{p}$ is called mean-type if $f=\left(M_{1}, \ldots, M_{p}\right)$, where each $M_{i}: I^{p} \rightarrow \mathbb{R}$ is a mean on $I$; that is, for each $\left(x_{1}, \ldots, x_{p}\right) \in I^{p}$,

$$
\min \left\{x_{1}, \ldots, x_{p}\right\} \leq M_{i}\left(x_{1}, \ldots, x_{p}\right) \leq \max \left\{x_{1}, \ldots, x_{p}\right\} .
$$


Notice that $f$ may not be continuous in general. However, if $f$ satisfies

$$
\left|M_{i}\left(x_{1}, \ldots, x_{p}\right)-z_{i}\right| \leq \max \left\{\left|x_{1}-z_{1}\right|, \ldots,\left|x_{p}-z_{p}\right|\right\}
$$

for all $\left(x_{1}, \ldots, x_{p}\right) \in I^{p},\left(z_{1}, \ldots, z_{p}\right) \in F(f)$ and $i=$ $1, \ldots, p$, it is always quasi-nonexpansive (with respect to the maximum norm), and hence uniformly virtually stable (with respect to the sequence $(n))$. For if $\left(x_{1}, \ldots, x_{p}\right) \in I^{p}$ and $\left(z_{1}, \ldots, z_{p}\right) \in F(f)$, one has

$$
\begin{aligned}
& \left\|f\left(x_{1}, \ldots, x_{p}\right)-f\left(z_{1}, \ldots, z_{p}\right)\right\| \\
& \quad=\max \left\{\left|M_{i}\left(x_{1}, \ldots, x_{p}\right)-z_{i}\right|: i=1, \ldots, p\right\} \\
& \quad \leq \max \left\{\left|x_{1}-z_{1}\right|, \ldots,\left|x_{p}-z_{p}\right|\right\} \quad[\operatorname{by}(*)] \\
& \quad=\left\|\left(x_{1}, \ldots, x_{p}\right)-\left(z_{1}, \ldots, z_{p}\right)\right\| .
\end{aligned}
$$

In particular, the mean-type mapping $f:(0, \infty)^{2} \rightarrow(0$, $\infty)^{2}$ defined by $f(x, y)=((x+y) / 2,2 x y /(x+y))$ satisfies $(*)$, and hence it is uniformly virtually stable. Since $f$ is also continuous, $F(f)$ is a retract of $C(f)$ by the previous theorem. In fact, it follows from [4] that $F(f)=\{(x, x): x>0\}, C(f)=$ $(0, \infty)^{2}$, and $f^{\infty}(x, y)=(\sqrt{x y}, \sqrt{x y})$ for $(x, y) \in(0, \infty)^{2}$. Therefore, $F(f)$ is clearly a retract of $C(f)$.

Example 5. Consider that $\mathbb{R}^{2}$ equipped with the maximum norm, and a discontinuous self-map $f: \mathbb{R}^{2} \rightarrow \mathbb{R}^{2}$ given by

$$
f(x, y)= \begin{cases}(0,1) & \text { if }(x, y)=(0,3) ; \\ (x,|x|) & \text { otherwise. }\end{cases}
$$

It is straightforward to verify that $f$ is quasi-nonexpansive (hence, uniformly virtually stable) and $f^{2}$ is continuous. Therefore, by the previous theorem, $f^{\infty}$ is continuous and hence $F(f)$ is a retract of $C(f)$. Notice also that $F(f)=$ $\{(x,|x|): x \in \mathbb{R}\}$ is not convex.

The following examples show that if we drop the continuity assumption of some iterate $f^{k}$ in Theorem 3, the map $f^{\infty}$ may or may not be continuous.

Example 6. Let $f:[0,1] \rightarrow[0,1]$ be defined by

$$
f(x)= \begin{cases}x & \text { if } x<\frac{1}{2} \\ 1 & \text { otherwise }\end{cases}
$$

Observe that $f$ is uniformly virtually stable and $f^{n}=f$ is not continuous for all $n \in \mathbb{N}$. Moreover, $F(f)=[0,1 / 2) \cup\{1\}$ and $C(f)=[0,1]$. Hence $f^{\infty}$ is not continuous.

Example 7. Consider that $\mathbb{R}^{2}$ equipped with the supremum norm. Define $f: \mathbb{R}^{2} \rightarrow \mathbb{R}^{2}$ by

$$
f(x, y)= \begin{cases}\left(0,3^{n-1}\right) & \text { if }(x, y)=\left(0,3^{n}\right) \text { for some } n \in \mathbb{N} \\ (x,|x|) & \text { otherwise. }\end{cases}
$$

It is straightforward to verify that $f$ is quasi-nonexpansive (hence, uniformly virtually stable) and $f^{n}$ is not continuous for all $n \in \mathbb{N}$. However, $f^{\infty}$ is continuous because $f^{\infty}(x, y)=$ $(x,|x|)$ for all $(x, y) \in \mathbb{R}^{2}$.

The next theorem gives a condition allowing us to enlarge the sequence associated with a given uniformly virtually stable self-map. As a result, we immediately obtain a refinement and a generalization of [1, Theorem 2.17].

Definition 8. An increasing sequence $\left(a_{n}\right)$ of natural numbers is said to satisfy the sup-finite condition if $\sup _{n}\left(a_{n+1}-a_{n}\right):=$ $\sup \left\{a_{n+1}-a_{n}: n \in \mathbb{N}\right\}<\infty$.

Theorem 9. If, for some $k \in \mathbb{N}, f^{k}$ is continuous on $F(f)$ and $f$ is uniformly virtually stable with respect to $\left(a_{n} k\right)$ where $\left(a_{n}\right)$ satisfies the sup-finite condition, then $f$ is uniformly virtually stable with respect to $(n k)$.

Proof. Let $s=\sup _{n}\left(a_{n+1}-a_{n}\right), p \in F(f)$, and $U \in \mathcal{N}(p)$. Because $f$ is uniformly virtually stable with respect to $\left(a_{n} k\right)$, there is $W \in \mathcal{N}(p)$ such that $f^{a_{n} k}(W) \subseteq U$ for all $n \in \mathbb{N}$. By continuity at $p$ of $f^{i k}, i \in \mathbb{N} \cup\{0\}$, there exists $V \in \mathcal{N}(p)$ for which $f^{i k}(V) \subseteq W \cap U$ for all $i=0, \ldots, \max \left\{s, a_{1}\right\}$. Now let $n \in \mathbb{N}$. If $n \leq a_{1}, f^{n k}(V) \subseteq W \cap U \subseteq U$, whereas if $n>a_{1}$, there is $j \in \mathbb{N}$ such that $n=a_{j}+i$ for some $i=0, \ldots, s$ and so

$$
\begin{aligned}
f^{n k}(V) & =f^{a_{j} k}\left(f^{i k}(V)\right) \subseteq f^{a_{j} k}(W \cap U) \\
& \subseteq f^{a_{j} k}(W) \subseteq U .
\end{aligned}
$$

Corollary 10. Suppose that $X$ is a metric space. If $f$ is continuous and uniformly virtually stable with respect to $\left(a_{n}\right)$, where $\left(a_{n}\right)$ satisfies the sup-finite condition, then $f$ is virtually nonexpansive. In particular, $C(f)$ is a $G_{\delta}$-set when $X$ is complete.

Proof. By setting $k=1$ in the previous theorem, $f$ is uniformly virtually stable with respect to $(n)$ and hence virtually nonexpansive by Proposition $2(1)$. For, if $X$ is complete, by [9, Theorem 1.2], $C(f)$ is then a $G_{\delta}$-set.

Corollary 11. Suppose that $X$ is a complete metric space. If, for some $k \in \mathbb{N}, f$ is uniformly virtually stable with respect to $(n k)$ and $f^{k}$ is continuous, then $C\left(f^{k}\right)$ is a $G_{\delta}$-set.

Proof. Let $g=f^{k}$. Then $g$ is uniformly virtually stable with respect to $(n)$. Thus, $C\left(f^{k}\right)=C(g)$ is a $G_{\delta}$-set by the previous corollary.

\section{Sequences Associated with Uniformly Virtually Stable Self-Maps}

In view of Proposition 2 (2) and Theorem 9, the sequence $\left(a_{n}\right)$ associated with a given uniformly virtually stable self-map $f$ seems to have an implicit structure. In fact, some questions may naturally arise. 
(i) Can we replace $\left(a_{n}\right)$ with the larger (in some sense) sequence $\left(a_{n}^{\prime}\right)$ so that $f$ is still uniformly virtually stable with respect to $\left(a_{n}^{\prime}\right)$ ?

(ii) If $\left(a_{n}\right)$ can be enlarged to $\left(a_{n}^{\prime}\right)$, can we make $\left(a_{n}^{\prime}\right)$ largest possible? and how does it look like?

(iii) Does the largest possible sequence $\left(a_{n}^{\prime}\right)$ satisfy the supfinite condition?

(iv) Is the largest possible sequence $\left(a_{n}^{\prime}\right)$ always $(n)$ ?

As we will see later on in this section, it turns out that the answers to these questions are interestingly nontrivial and require some careful considerations in both continuous and discontinuous settings.

Throughout this section, $X$ is a Hausdorff space and $f$ is a self-map of $X$. Every sequence is assumed to be an increasing sequence of natural numbers, so it is uniquely determined by its image. We then always identify a sequence $\left(a_{n}\right)$ with the infinite set $\left\{a_{n}: n \in \mathbb{N}\right\}$, and with this identification in mind, the following notations become natural:

(i) $\left(a_{n}\right) \subseteq\left(b_{n}\right)$ if $\left(a_{n}\right)$ is a subsequence of $\left(b_{n}\right)$;

(ii) $\left(a_{n}\right) \cup\{c\}$ represents the sequence whose image is $\left\{a_{n}\right.$ : $n \in \mathbb{N}\} \cup\{c\}$;

(iii) $\left(a_{n}+c\right)$ represents the sequence whose image is $\left\{a_{n}+c\right.$ : $n \in \mathbb{N}\}$

(iv) $\left(a_{n}\right) \cup\left(b_{n}\right)$ represents the sequence whose image is $\left\{a_{n}: n \in \mathbb{N}\right\} \cup\left\{b_{n}: n \in \mathbb{N}\right\}$, and so $\left(a_{n}\right) \cup \cdots \cup\left(b_{n}\right)$ represents the sequence whose image is $\left\{a_{n}: n \in \mathbb{N}\right\} \cup$ $\cdots \cup\left\{b_{n}: n \in \mathbb{N}\right\}$;

(v) $\left(a_{n}\right) \oplus\left(b_{n}\right)$ represents the sequence whose image is $\left\{a_{i}+\right.$ $\left.b_{j}: i, j \in \mathbb{N}\right\}$, and $\oplus_{i=1}^{k}\left(a_{n}\right)=\underbrace{\left(a_{n}\right) \oplus \cdots \oplus\left(a_{n}\right)}_{k \text { copies of }\left(a_{n}\right)}$.

For a nonempty subset $A$ of $\mathbb{N}$, the natural number $d$ satisfying

(1) $d \mid a$ for all $a \in A$ and

(2) if $c \mid a$ for all $a \in A$, then $d \mid c$

will be called the greatest common divisor of $A$ and denoted by $\operatorname{gcd} A$. Also, we let $\langle A\rangle$ denote the set of all finite sums of elements in $A$; that is,

$$
\langle A\rangle=\left\{x: x=x_{1}+\cdots+x_{k} \text { for some } x_{1}, \ldots, x_{k} \in A\right\} .
$$

Notice that $A \subseteq\langle A\rangle$ and if $1 \in A$, we have $\langle A\rangle=\mathbb{N}$. As usual, $\operatorname{gcd}\left(a_{n}\right)$ will represent $\operatorname{gcd}\left\{a_{n}: n \in \mathbb{N}\right\}$, and $\left\langle a_{n}\right\rangle$ will represent at the same time both the set $\left\langle\left\{a_{n}: n \in \mathbb{N}\right\}\right\rangle$ and the sequence whose image is $\left\langle\left\{a_{n}: n \in \mathbb{N}\right\}\right\rangle$. We may simply write $\left\langle a_{1}, \ldots, a_{n}\right\rangle$ for $\left\langle\left\{a_{1}, \ldots, a_{n}\right\}\right\rangle$. let

Moreover, for a uniformly virtually stable self-map $f$, we

$$
\begin{gathered}
\mathcal{S}_{f}=\left\{\left(a_{n}\right): f\right. \text { is uniformly virtually stable } \\
\text { with respect to } \left.\left(a_{n}\right)\right\} .
\end{gathered}
$$

Clearly, $\delta_{f}$ is partially ordered by $\subseteq$, and a maximal element in $\left(\mathcal{S}_{f}, q\right.$ will be called a maximal sequence associated with $f$. The following are some basic properties of $\mathcal{S}_{f}$.
Proposition 12. Suppose $f$ is uniformly virtually stable with respect to $\left(a_{n}\right)$. one has the following:

(1) If $\left(b_{n}\right) \subseteq\left(a_{n}\right)$, then $\left(b_{n}\right) \in \mathcal{S}_{f}$.

(2) If $\left(b_{n}\right) \in \mathcal{S}_{f}$, then $\left(a_{n}\right) \cup\left(b_{n}\right) \in \mathcal{S}_{f}$.

(3) $\oplus_{i=1}^{k}\left(a_{n}\right) \in \mathcal{S}_{f}$ for any $k \in \mathbb{N}$.

(4) $\left(a_{n}+c\right),\left(a_{n}\right) \cup\{c\} \in \mathcal{S}_{f}$ for any $c \in\left\langle a_{n}\right\rangle$.

Proof. Let $x \in F(f)$ and $U \in \mathcal{N}(x)$.

(1) It is straightforward from the definition of virtual stability.

(2) There exist $V, W \in \mathscr{N}(x)$ such that $f^{a_{n}}(V) \subseteq U$ and $f^{b_{n}}(W) \subseteq U$ for all $n \in \mathbb{N}$. Hence $f^{c_{n}}(V \cap W) \subseteq U$ for all $n \in \mathbb{N}$ where $\left(c_{n}\right)=\left(a_{n}\right) \cup\left(b_{n}\right)$.

(3) By induction, it suffices to show that $\left(a_{n}\right) \oplus\left(a_{n}\right) \in \mathcal{S}_{f}$. There are, by virtual stability of $f, V, W \in \mathcal{N}(x)$ such that $f^{a_{n}}(V) \subseteq U$ and $f^{a_{n}}(W) \subseteq V$ for all $n$. This implies that $f^{a_{n}+a_{m}}(W) \subseteq f^{a_{n}}(V) \subseteq U$ for all $n, m \in \mathbb{N}$.

(4) By letting $c \in\left\langle a_{n}\right\rangle$, we have $c=a_{i_{1}}+\cdots+a_{i_{k}} \in \oplus_{i=1}^{k}\left(a_{n}\right)$ for some $k \in \mathbb{N}$, and hence, $\left(a_{n}+c\right) \subseteq \oplus_{i=1}^{k+1}\left(a_{n}\right) \in \mathcal{S}_{f}$ and $\left(a_{n}\right) \cup\{c\} \subseteq \bigcup_{j=1}^{k} \oplus_{i=1}^{j}\left(a_{n}\right) \in \mathcal{S}_{f}$ by (1) and (2).

Remark 13. From (2) in the previous proposition, if $\left(a_{n}\right)$ and $\left(b_{n}\right)$ are maximal elements in $\left(\mathcal{S}_{f}, \subseteq\right)$, then $\left(a_{n}\right)=\left(a_{n}\right) \cup\left(b_{n}\right)=$ $\left(b_{n}\right)$. This implies that a maximal sequence associated with $f$, if exists, is always unique.

Before considering the existence, we will first investigate the structure of the maximal sequence associated with a given self-map.

Lemma 14. Suppose $f$ is uniformly virtually stable. For a sequence $\left(a_{n}\right)$, one has

(1) $\operatorname{gcd}\left(a_{n}\right)=\operatorname{gcd}\left\langle a_{n}\right\rangle=\operatorname{gcd}\left\{a_{i}: i \leq m\right\}$ for some $m \in \mathbb{N}$,

(2) there are $b \in\left\langle a_{n}\right\rangle$ and $m \in \mathbb{N}$ such that $k \operatorname{gcd}\left(a_{n}\right)+b \in$ $\left\langle a_{1}, \ldots, a_{m}\right\rangle$ for all $k \geq 0$,

(3) if $\left(a_{n}\right) \in \mathcal{S}_{f}$ and $\left(a_{n}\right)$ satisfies the sup-finite condition, then $\left\langle a_{n}\right\rangle \in \mathcal{S}_{f}$.

Proof. (1) The fact that $\operatorname{gcd}\left(a_{n}\right)=\operatorname{gcd}\left\langle a_{n}\right\rangle$ follows directly from the definition. Now, let $d=\min \left\{\operatorname{gcd}\left\{a_{i}: i \leq k\right\}: k \in \mathbb{N}\right\}$. Then, $d=\operatorname{gcd}\left\{a_{i}: i \leq m\right\}$ for some $m \in \mathbb{N}$. If $d+a_{n}$ for some $n>m$, then $\operatorname{gcd}\left\{a_{1}, \ldots, a_{n}\right\}<d$, a contradiction. Also, for any $c>0$ satisfying $c \mid a_{n}$ for all $n \in \mathbb{N}$, we have $c \mid a_{i}$ for all $1 \leq i \leq m$, and hence, $c \leq d$. This implies $\operatorname{gcd}\left(a_{n}\right)=\operatorname{gcd}\left\{a_{i}: i \leq m\right\}$.

(2) If $\operatorname{gcd}\left(a_{n}\right)=a_{1}$, the proof is clear. So, we assume that $\operatorname{gcd}\left(a_{n}\right) \neq a_{1}$. By $(1), \operatorname{gcd}\left(a_{n}\right)=\operatorname{gcd}\left\{a_{i}: i \leq m\right\}$ for some $m \in \mathbb{N}$. Then, for some partition $\{P, N\}$ of $\{1, \ldots, m\}$, $\operatorname{gcd}\left(a_{n}\right)=\sum_{i \in P} n_{i} a_{i}-\sum_{i \in N} n_{i} a_{i}$ where $n_{i} \geq 0$. Set $A=\sum_{i \in P} n_{i} a_{i}$ and $B=\sum_{i \in N} n_{i} a_{i}$. Notice that $B \neq 0$ because $\operatorname{gcd}\left(a_{n}\right) \neq a_{1}$, 
and $l B \in\left\langle a_{1}, \ldots, a_{m}\right\rangle$ for all $l \in \mathbb{N}$. Then for each $l \in \mathbb{N}$ and $0 \leq r \leq B$,

$$
r \operatorname{gcd}\left(a_{n}\right)+B^{2}+l B=r A+(B-r+l) B \in\left\langle a_{1}, \ldots, a_{m}\right\rangle .
$$

Since for $k \geq 0$, we have $k=q B+r$ for some $q \geq 0$ and $0 \leq r<B$,

$$
\begin{aligned}
& k \operatorname{gcd}\left(a_{n}\right)+\left(B^{2}+B\right) \\
& \quad=r \operatorname{gcd}\left(a_{n}\right)+B^{2}+\left(1+q \operatorname{gcd}\left(a_{n}\right)\right) B \in\left\langle a_{1}, \ldots, a_{m}\right\rangle .
\end{aligned}
$$

Therefore, the result follows by letting $b=B^{2}+B$.

(3) Assume that $\left(a_{n}\right) \in \mathcal{S}_{f}$ and $\Delta:=\sup _{n}\left(a_{n+1}-a_{n}\right)<\infty$. By (2), there is $b \in\left\langle a_{n}\right\rangle$ for which $k \operatorname{gcd}\left(a_{n}\right)+b \in\left\langle a_{n}\right\rangle$ for all $k \geq 0$. By Proposition 12, we have

$$
\begin{aligned}
\left(b_{n}\right):= & \left(a_{n}\right) \cup\left(a_{n}+\operatorname{gcd}\left(a_{n}\right)+b\right) \\
& \cup\left(a_{n}+2 \operatorname{gcd}\left(a_{n}\right)+b\right) \\
& \cup \cdots \cup\left(a_{n}+\Delta \operatorname{gcd}\left(a_{n}\right)+b\right) \in \mathcal{S}_{f},
\end{aligned}
$$

and hence $\left(c_{n}\right):=\left(b_{n}\right) \cup\left\{c \in\left\langle a_{n}\right\rangle: c \leq a_{1}+b\right\} \in \mathcal{S}_{f}$. The proof will immediately follow by Proposition 12 (1) once we can show that $\left\langle a_{n}\right\rangle \subseteq\left(c_{n}\right)$. To see this, let $c \in\left\langle a_{n}\right\rangle$. The case of $c \leq a_{1}+b$ is clear. Otherwise, $a_{m}+b \leq c<a_{m+1}+b$ for some $m \geq 1$. Since $\operatorname{gcd}\left(a_{n}\right) \mid c-a_{m}-b$, we have $c=$ $a_{m}+b+r \operatorname{gcd}\left(a_{n}\right)$ for some $0 \leq r<a_{m+1}-a_{m} \leq \Delta$. This implies that $c \in\left(a_{n}+r \operatorname{gcd}\left(a_{n}\right)+b\right) \subseteq\left(b_{n}\right) \subseteq\left(c_{n}\right)$.

Theorem 15. Suppose $f$ is uniformly virtually stable and $\left(a_{n}\right)$ is the maximal sequence associated with $f$. One has

(1) $\left(a_{n}\right)=\left\langle a_{n}\right\rangle$,

(2) $\left(a_{n}\right)=\langle A\rangle$ for some finite subset $A$ of $\left(a_{n}\right)$,

(3) if $f$ is continuous, then $\left(a_{n}\right)=(n)$.

Proof. (1) It suffices to show that $\left\langle a_{n}\right\rangle \subseteq\left(a_{n}\right)$. Let $c \in\left\langle a_{n}\right\rangle$. By Proposition 12, $\left(a_{n}\right) \cup\{c\} \in \mathcal{S}_{f}$, and hence, $c \in\left(a_{n}\right)$ by maximality of $\left(a_{n}\right)$.

(2) From (1), we have $\left(a_{n}\right)=\left\langle a_{n}\right\rangle$. Then by Lemma 14 (2), there exist $l, m \in \mathbb{N}$ such that $k \operatorname{gcd}\left(a_{n}\right)+a_{l} \in\left\langle a_{1}, \ldots, a_{m}\right\rangle$ for all $k \geq 0$. By setting $A=\left\{a_{i}: i \leq \max \{l, m\}\right\}$, we clearly have $\langle A\rangle \subseteq\left\langle a_{n}\right\rangle=\left(a_{n}\right)$. On the other hand, for each $i \in \mathbb{N}$, notice that

(i) if $i \leq \max \{l, m\}$, then $a_{i} \in A$, and

(ii) if $i>\max \{l, m\}$, then $a_{i}=a_{l}+k \operatorname{gcd}\left(a_{n}\right) \in$ $\left\langle a_{1}, \ldots, a_{m}\right\rangle \subseteq\langle A\rangle$ for some $k \in \mathbb{N}$.

Therefore, $\left(a_{n}\right) \subseteq\langle A\rangle$.

(3) Suppose that $f$ is continuous. It follows that $\left(a_{n}\right) \cup\{1\} \in$ $\mathcal{S}_{f}$, and by maximality of $\left(a_{n}\right), 1 \in\left(a_{n}\right)$. Therefore, by (1), we have $\left(a_{n}\right)=\left\langle a_{n}\right\rangle=(n)$.

The next theorem gives a necessary and sufficient condition for the existence of the maximal sequence.

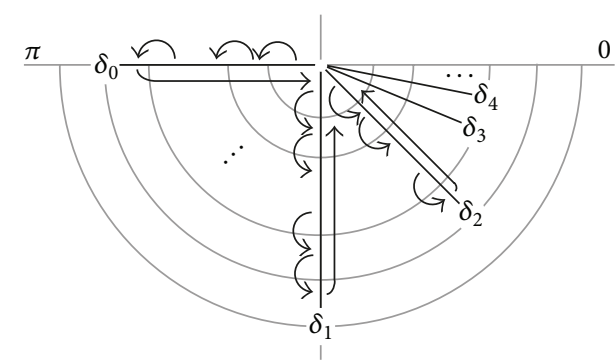

Figure 1: The map $f$ in Example 17.

Theorem 16. Suppose $f$ is uniformly virtually stable. Then the maximal sequence associated with $f$ exists if and only if there is $\left(a_{n}\right) \in \mathcal{S}_{f}$ satisfying the sup-finite condition.

Proof. $(\Rightarrow)$ If $\left(a_{n}\right)$ is maximal, then $\left(n a_{1}\right) \subseteq\left(a_{n}\right)$ and hence

$$
\sup _{n}\left(a_{n+1}-a_{n}\right) \leq \sup _{n}\left((n+1) a_{1}-n a_{1}\right)=a_{1}<\infty .
$$

$(\Leftarrow)$ Assume that $\Delta:=\sup _{n}\left(a_{n+1}-a_{n}\right)<\infty$ for some $\left(a_{n}\right) \epsilon$ $\mathcal{S}_{f}$. By Lemma $14(3),\left\langle a_{n}\right\rangle \in \mathcal{S}_{f}$. If $a_{1}=1$, we are done. Suppose that $a_{1}>1$. For $0 \leq i<a_{1}=\min \left\langle a_{n}\right\rangle$, let

$$
A_{i}=\left\{m: m \equiv i \bmod a_{1} \text { and }\left\langle a_{n}\right\rangle \cup\{m\} \in \mathcal{S}_{f}\right\},
$$

and $m_{i}=\min A_{i}$ if $A_{i} \neq \emptyset$, otherwise $m_{i}=a_{1}$. Then $\left\{c:\left\langle a_{n}\right\rangle \cup\right.$ $\left.\{c\} \in \mathcal{S}_{f}\right\}=A_{0} \cup \cdots \cup A_{a_{1}-1}$. By Proposition $12(2),\left\langle a_{n}\right\rangle \cup\left\{m_{i}\right.$ : $\left.0 \leq i<a_{1}\right\} \in \mathcal{S}_{f}$ and again by Lemma 14 (3), since $\sup _{n}\left(b_{n+1}-\right.$ $\left.b_{n}\right) \leq \sup _{n}\left(a_{n+1}-a_{n}\right)<\infty$ where $\left(b_{n}\right):=\left\langle\left\langle a_{n}\right\rangle \cup\left\{m_{i}: 0 \leq i<\right.\right.$ $\left.\left.a_{1}\right\}\right\rangle,\left(b_{n}\right) \in \mathcal{S}_{f}$. Notice that $\left(b_{n}\right)$ is maximal because whenever $\left(b_{n}\right) \cup\{c\} \in \mathcal{S}_{f}$ for some $c$, there is $0 \leq i<a_{1}$ such that $c \in A_{i} \subseteq A_{0} \cup \cdots \cup A_{a_{1}-1} \subseteq\left(b_{n}\right)$.

Towards the end of this section, we will construct some interesting examples including a uniformly virtually stable self-map whose maximal sequence is prescribed as well as a uniformly virtually stable self-map having no maximal sequence.

Example 17. For $k \in \mathbb{N} \cup\{0\}$, let $\theta_{k}=\pi \sum_{i=0}^{k}\left(1 / 2^{i}\right)$. Then $\pi \leq \theta_{k}<2 \pi$ for all $k$. Consider the sequence $\left(a_{n}\right)=\left\langle a_{n}\right\rangle$. Set $\delta_{0}=a_{1}$ and $\delta_{n}=a_{n+1}-a_{n}$ for any $n \in \mathbb{N}$. Define $f: \mathbb{C} \rightarrow \mathbb{C}$ by

$$
\begin{aligned}
& f(z)= f\left(r e^{i \phi}\right) \\
&= \begin{cases}(r+1) e^{i \phi} & \text { if } \phi=\theta_{k}, 0<r<\delta_{k} \\
(r-\lfloor r\rfloor) e^{i(\pi+\phi / 2)} & \text { for some } k ; \\
0 & \text { if } \phi=\theta_{k}, r \geq \delta_{k}-1\end{cases} \\
& \text { for some } k ;
\end{aligned}
$$

Then, $f$ can be illustrated in Figure 1 and it is uniformly virtually stable with respect to the maximal sequence $\left(a_{n}\right)$. 
To see this, note that $F(f)=\{0\}, \pi+\theta_{k} / 2=\pi(1+$ $\left.\sum_{i=1}^{k+1}\left(1 / 2^{i}\right)\right)=\pi \sum_{i=0}^{k+1}\left(1 / 2^{i}\right)=\theta_{k+1}$ for all $k \geq 0$, and for each $n \in \mathbb{N}$ and $0<r<1$,

$$
\begin{aligned}
f^{a_{n}}\left(r e^{i \theta_{0}}\right) & =f^{\delta_{n-1}+\cdots+\delta_{0}}\left(r e^{i \theta_{0}}\right) \\
& =f^{\delta_{n-1}+\cdots+\delta_{1}}\left(r e^{i \theta_{1}}\right)=\cdots=r e^{i \theta_{n}} .
\end{aligned}
$$

To show the virtual stability of $f$ with respect to $\left(a_{n}\right)$, it suffices to prove that $f^{a_{n}}(B(0 ; r)) \subseteq B(0 ; r)$ for all $0<r<1$. Let $0<r<1$. For each $z=s e^{i \phi} \in B(0 ; r)$,

$$
\begin{aligned}
\left|f^{a_{n}}(z)\right| & \leq\left|f^{a_{n}}\left(s e^{i \theta_{k}}\right)\right|=\left|f^{a_{n}} f^{a_{k}}\left(s e^{i \theta_{0}}\right)\right| \\
& =\left|f^{a_{m}}\left(s e^{i \theta_{0}}\right)\right|=\left|s e^{i \theta_{m}}\right|=s<r
\end{aligned}
$$

for some $k$, and $a_{m}=a_{n}+a_{k}$. Thus, we get the claim. Moreover $\left(a_{n}\right)$ is maximal because whenever $b \notin\left(a_{n}\right)$, either $b<a_{1}$ or $a_{m}<b<a_{m+1}$ for some $m \in \mathbb{N}$ (and so $b=a_{m}+k$ for some $\left.1 \leq k<\delta_{m}\right)$ holds, which the first case implies that $f^{b}\left(r e^{i \theta_{0}}\right)=(r+b) e^{i \theta_{0}} \notin B(0 ; 1)$ for all $0<r<1$, while the latter implies that

$$
\begin{aligned}
\left|f^{b}\left(r e^{i \theta_{0}}\right)\right| & =\left|f^{k} f^{a_{m}}\left(r e^{i \theta_{0}}\right)\right| \\
& =\left|f^{k}\left(r e^{i \theta_{m}}\right)\right|=\left|(r+k) e^{i \theta_{m}}\right| \\
& =r+k>1
\end{aligned}
$$

for all $0<r<1$; that is, $f^{b}\left(r e^{i \theta_{0}}\right) \notin B(0 ; 1)$. Thus $\left(a_{n}\right) \cup\{b\} \notin$ $\mathcal{S}_{f}$ for all $b \notin\left(a_{n}\right)$, which induces the maximality of $\left(a_{n}\right)$ as desired.

Lemma 18. Let $A_{1}=\left\{2^{n}: n \in \mathbb{N}\right\}$ and for $m>1$,

$$
A_{m}=\left\{2^{n_{1}}+\cdots+2^{n_{m}}: n_{i} \in \mathbb{N}\right\}-\bigcup_{i<m} A_{i} .
$$

Then $\left\{A_{n}: n \in \mathbb{N}\right\}$ forms a partition of $2 \mathbb{N}:=\{2 n: n \in \mathbb{N}\}$, and $A_{n}$ is infinite for each $n \in \mathbb{N}$.

Proof. Following from the fact that $2^{n}+2^{n}=2^{n+1}$ for all $n \in$ $\mathbb{N}$, if $\sum_{i=1}^{m} 2^{n_{i}} \in A_{m}$, then $n_{i} \neq n_{j}$ for all $i \neq j$. Also for each $n \in \mathbb{N}$, since $2 n=\sum_{i=1}^{n} 2 \in \bigcup_{i=1}^{n} A_{i}$, we have $2 n \in A_{i}$ for some $i \leq n$. Hence, $\left\{A_{n}: n \in \mathbb{N}\right\}$ forms a partition of $2 \mathbb{N}$. For the next implication, notice that $A_{1}$ is clearly infinite. Now, assume that $n>1$. We first claim that $2^{n}-2 \in A_{n-1}$. Since $2^{n}-2 \in 2 \mathbb{N}$, we have $2^{n}-2 \in A_{k}$ for some $k \in \mathbb{N}$. It follows that $k<n$ and for if $2^{n}-2=\sum_{i=1}^{k} 2^{n_{i}}$, then $n_{i}<n$ and $\sum_{i=1}^{k} 2^{n_{i}}=$ $2^{n}-2=\sum_{i=1}^{n-1} 2^{i}$, and hence $k=n-1$. Next, we claim that $2^{n+m}+2^{n}-2 \in A_{n}$ for all $m \in \mathbb{N}$. Let $m \in \mathbb{N}$. By the previous claim, we have $2^{n+m}+\left(2^{n}-2\right) \in \bigcup_{i=1}^{n} A_{i}$. Hence, $2^{n+m}+2^{n}-2 \in$ $A_{k}$ for some $1 \leq k \leq n$; that is, $2^{n+m}+2^{n}-2=\sum_{i=1}^{k} 2^{n_{i}}$. Since $\sum_{i=1}^{n+m-1} 2^{i}=2^{n+m}-2<2^{n+m}+2^{n}-2<2^{n+m+1}$, we must have $2^{n_{i}}=2^{n+m}$ for some $1 \leq i \leq k$. Thus, $2^{n+m}+2^{n}-2 \in A_{n}$ as claimed. Finally, since $\left\{2^{n+m}+2^{n}-2: m \in \mathbb{N}\right\} \subseteq A_{n}$, then $A_{n}$ is infinite as desired.
Example 19. For each $m \in \mathbb{N}$, let $A_{m}$ be defined as in the previous lemma, $p_{1}=3$ and for each $n>1$, let $p_{n}$ be the smallest prime number such that $p_{n}>\max \left\{n^{2}+n, p_{n-1}\right\}$. For examples, $p_{2}=7$ and $p_{3}=13$. Clearly, $p_{n} \neq p_{m}$ whenever $n \neq m$. Now, let $X=[0,2] \times[0,2]$ be a subspace of $\mathbb{R}^{2}$ equipped with the maximum norm, $0:=(0,0) \in X$ and let $B(a ; r)$ denote the open ball $\{x \in X:\|x-a\|<r\}$.

The goal of this example is to find a uniformly virtually stable self-map having no maximal sequence. This can be done by constructing a uniformly virtually stable self-map $f: X \rightarrow X$ with respect to $\left(2^{n}\right)$ but not $(2 n)$. For if $\left(a_{n}\right)$ is the maximal sequence associated with such an $f$, we must have $\left(2^{n}\right) \subseteq\left(a_{n}\right)$ and hence $(2 n)=\left\langle 2^{n}\right\rangle \subseteq\left\langle a_{n}\right\rangle=\left(a_{n}\right)$, but this contradicts the property that $f$ is not uniformly virtually stable with respect to $(2 n)$.

To obtain the desired self-map, it suffices to require the map $f: X \rightarrow X$ to satisfy the following three conditions:

(C1) $F(f)=\{\mathbf{0}\}$

(C2) $f^{2^{n}}(B(\mathbf{0} ; 1 /(i+1))) \subseteq B(\mathbf{0} ; 1 / i)$ for each $i, n \in \mathbb{N}$,

(C3) For each $k \in \mathbb{N}$, there exists $a \in 2 \mathbb{N}$ such that $f^{a}(1 /(k+$ $2), 0) \notin B(\mathbf{0} ; 1)$.

Notice that (C1) and (C2) imply the virtual stability of $f$ with respect to $\left(2^{n}\right)$, while $(\mathrm{C} 1)$ and $(\mathrm{C} 3)$ imply that, for each $k \in \mathbb{N}$, there exists $a \in 2 \mathbb{N}$ such that $f^{a}(B(0 ; 1 /(k+1))) \nsubseteq$ $B(0 ; 1)$, and hence $f$ is not uniformly virtually stable with respect to $(2 n)$.

We are now ready to give the explicit description of such an $f$. Consider the following subsets of $X$ :

$$
\begin{aligned}
& T_{1}=\{(1 /(k+2), 0): k \in \mathbb{N}\}, \\
& T_{2}=\left\{\left(1,1 / p_{k}^{l}\right): k, l \in \mathbb{N} \text { and } l+1 \in A_{m}\right. \text { for some } \\
& m \leq k\}, \\
& T_{3}=\left\{\left(1 /(k+2-m), 1 / p_{k}^{l}\right): k, l \in \mathbb{N} \text { and } l \in A_{m}\right. \text { for } \\
& \text { some } m \leq k\}, \\
& T_{4}=\left\{\left(1,1 / p_{k}^{l}\right): k, l \in \mathbb{N a n d} \text { either } l \in A_{m} \text { or } l+1 \in\right. \\
& \left.A_{m} \text { for some } m>k\right\} .
\end{aligned}
$$

Observe that

(O1) $T_{i} \cap T_{j}=\emptyset$ whenever $i \neq j$. All cases are trivial except for the case of $T_{2} \cap T_{4}$, where it follows from the fact that $\left\{A_{m}: m \in \mathbb{N}\right\}$ forms a partition of $2 \mathbb{N}$;

(O2) $\|(x, y)\|=x$ for all $(x, y) \in \bigcup_{i=1}^{4} T_{i}$, because for each $k, m, l \in \mathbb{N}$ with $m \leq k$, we have $p_{k}^{l} \geq p_{k}>k^{2}+k \geq$ $k+1$ and hence $1 / p_{k}^{l}<1 /(k+1) \leq 1 /(k+2-m)$. 
Define $f: X \rightarrow X$ by

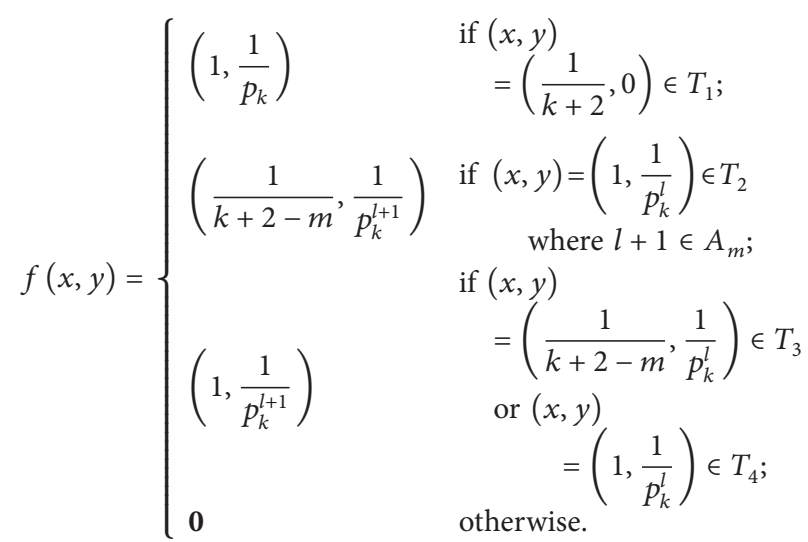

Clearly, $f$ is well-defined by (O1). The following properties are also satisfied.

(P1) $f\left(T_{1}\right) \subseteq T_{2}, f\left(T_{2}\right) \subseteq T_{3}$ and $f\left(T_{3} \cup T_{4}\right) \subseteq T_{2} \cup T_{4}$. So, $\bigcup_{i=1}^{4} T_{i}$ is $f$-invariant. Moreover, since $p_{i}$ 's are all distinct primes, we have $p_{i}^{k}=p_{j}^{l}$ if and only if $(i, k)=$ $(j, l)$, and hence $\left.\left(\pi_{2} \circ f\right)\right|_{\bigcup_{i=1}^{4} T_{i}}$ is injective, where $\pi_{2}$ denotes the second-coordinate projection $\pi_{2}(x, y)=$ $y$.

(P2) For each $k, l \in \mathbb{N}$, one can verify that

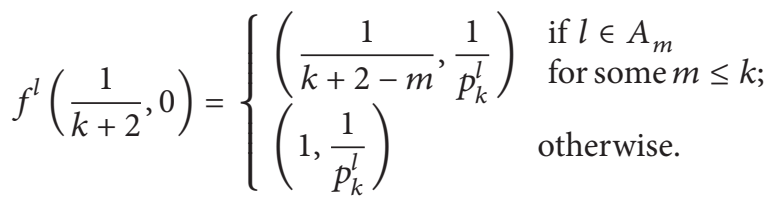

In particular, we have the following.

(i) $f^{2^{l}}(1 /(k+2), 0)=\left(1 /(k+2-1), 1 / p_{k}^{2^{l}}\right)=(1 /(k+$ $\left.1), 1 / p_{k}^{2^{l}}\right)$ since $2^{l} \in\left(2^{n}\right)=A_{1}$ and $1 \leq k$.

(ii) $f^{c}(1 /(k+2), 0)=\left(1,1 / p_{k}^{c}\right)$, where $c=\min A_{k+1}$, since $c \in A_{k+1}$ and $k+1>k$.

(P3) For each $k, l, m, n \in \mathbb{N}$ with $m<k$ and $l \in A_{m}$, we have $m+1 \leq k, f^{l}(1 /(k+2), 0)=\left(1 /(k+2-m), 1 / p_{k}^{l}\right)$ by (P2), and $2^{n}+l \in A_{j} \subseteq \bigcup_{i=1}^{m+1} A_{i}$ for some $j \leq m+1$. Again by (P2), it follows that

$$
\begin{aligned}
\left(\frac{1}{k+2-j}, \frac{1}{p_{k}^{2^{n}+l}}\right) & =f^{2^{n}+l}\left(\frac{1}{k+2}, 0\right) \\
& =f^{2^{n}}\left(\frac{1}{k+2-m}, \frac{1}{p_{k}^{l}}\right) .
\end{aligned}
$$

Clearly, (C1) is implied by (P1). To prove (C2), let $i, n \in \mathbb{N}$, and it suffices to consider the following two cases.
(1) If $(x, y)=(1 /(k+2), 0) \in T_{1} \cap B(0 ; 1 /(i+1))$, then $1 /(k+2)<1 /(i+1)$, and hence

$$
\begin{aligned}
\left\|f^{2^{n}}(x, y)\right\| & =\left\|f^{2^{n}}\left(\frac{1}{k+2}, 0\right)\right\| \\
& \stackrel{(\mathrm{P} 2)}{=}\left\|\left(\frac{1}{k+1}, \frac{1}{p_{k}^{2^{n}}}\right)\right\| \stackrel{(\mathrm{O} 2)}{=} \frac{1}{k+1}<\frac{1}{i} .
\end{aligned}
$$

(2) If $(x, y)=\left(1 /(k+2-m), 1 / p_{k}^{l}\right) \in T_{3} \cap B(\mathbf{0} ; 1 /(i+1))$, then $l \in A_{m}$ for some $m \leq k$, and $1 /(k+2-m) \stackrel{(\mathrm{O} 2)}{=}$ $\left\|\left(1 /(k+2-m), 1 / p_{k}^{l}\right)\right\|<1 /(i+1)$. If $m=k$, we have $1 / 2=1 /(k+2-m)<1 /(i+1)$, a contradiction. Thus $m<k$, and by (P3), there is some $j \leq m+1$ such that

$$
\begin{aligned}
&\left\|f^{2^{n}}\left(\frac{1}{k+2-m}, \frac{1}{p_{k}^{l}}\right)\right\| \stackrel{(\mathrm{P} 3)}{=}\left\|\left(\frac{1}{k+2-j}, \frac{1}{p_{k}^{2^{n}+l}}\right)\right\| \\
& \stackrel{(\mathrm{O} 2)}{=} \frac{1}{k+2-j} \\
& \leq \frac{1}{k+2-(m+1)}<\frac{1}{i} .
\end{aligned}
$$

From above cases, it follows that $f^{2^{n}}(B(0 ; 1 /(i+1))) \subseteq$ $B(\mathbf{0} ; 1 / i)$.

Finally, for (C3), let $k \in \mathbb{N}$ and $c=\min A_{k+1} \in 2 \mathbb{N}$. Then by $(\mathrm{P} 2)$, we have $f^{c}(1 /(k+2), 0)=\left(1,1 / p_{k}^{k+1}\right) \notin B(\mathbf{0} ; 1)$.

Remark 20. From the previous example, we can easily check that $f$ is uniformly virtually stable with respect to $\left(a_{n}\right)=A_{1} \cup$ $\cdots \cup A_{m}$ for any $m \in \mathbb{N}$, just by showing that $f^{a_{n}}(B(\mathbf{0} ; 1 /(k+$ $m))) \subseteq B(\mathbf{0} ; 1 / k)$ for any $k, n \in \mathbb{N}$. Hence, Proposition 12 (2) does not hold in general for an arbitrary union of sequences in $\mathcal{S}_{f}$.

\section{Acknowledgments}

The first author is (partially) supported by the Centre of Excellence in Mathematics, the Commission on Higher Education, Thailand. The third author wishes to thank the $\mathrm{H}$. M. King Bhumibol Adulyadej's 72nd Birthday Anniversary Scholarship. The authors are grateful to Professor Pimpen Vejjajiva and the anonymous referee(s) for their valuable comments and suggestions for improving this paper.

\section{References}

[1] P. Chaoha and W. Atiponrat, "Virtually stable maps and their fixed point sets," Journal of Mathematical Analysis and Applications, vol. 359, no. 2, pp. 536-542, 2009.

[2] P. Chaoha and P. Chanthorn, "Fixed point sets through iteration schemes," Journal of Mathematical Analysis and Applications, vol. 386, no. 1, pp. 273-277, 2012.

[3] J. Matkowski, "Invariant and complementary quasi-arithmetic means," Aequationes Mathematicae, vol. 57, no. 1, pp. 87-107, 1999. 
[4] J. Matkowski, "Iterations of mean-type mappings and invariant means," Annales Mathematicae Silesianae, no. 13, pp. 211-226, 1999.

[5] J. Matkowski, "Fixed points and iterations of mean-type mappings," Central European Journal of Mathematics, vol. 10, no. 6, pp. 2215-2228, 2012.

[6] T. Suzuki, "Fixed point theorems and convergence theorems for some generalized nonexpansive mappings," Journal of Mathematical Analysis and Applications, vol. 340, no. 2, pp. 1088-1095, 2008.

[7] P. Chaoha, "Virtually nonexpansive maps and their convergence sets," Journal of Mathematical Analysis and Applications, vol. 326, no. 1, pp. 390-397, 2007.

[8] R. Kannan, "Some results on fixed points-II," The American Mathematical Monthly, vol. 76, pp. 405-408, 1969.

[9] P. Chaoha and P. Chanthorn, "Convergence and fixed point sets of generalized homogeneous maps," Thai Journal of Mathematics, vol. 5, no. 2, pp. 281-289, 2007. 


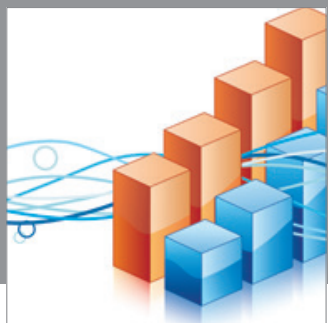

Advances in

Operations Research

mansans

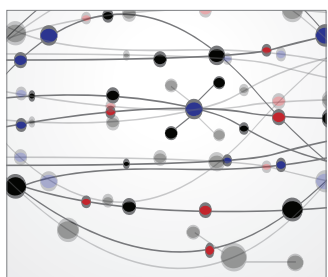

The Scientific World Journal
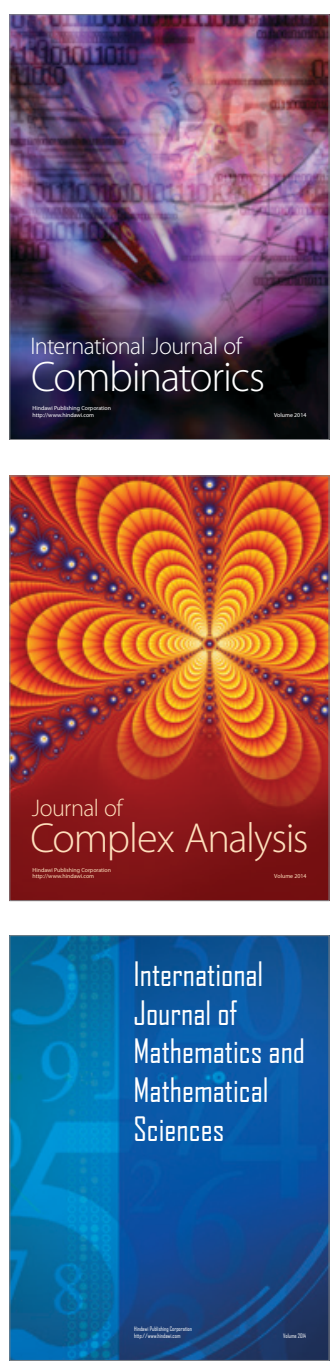
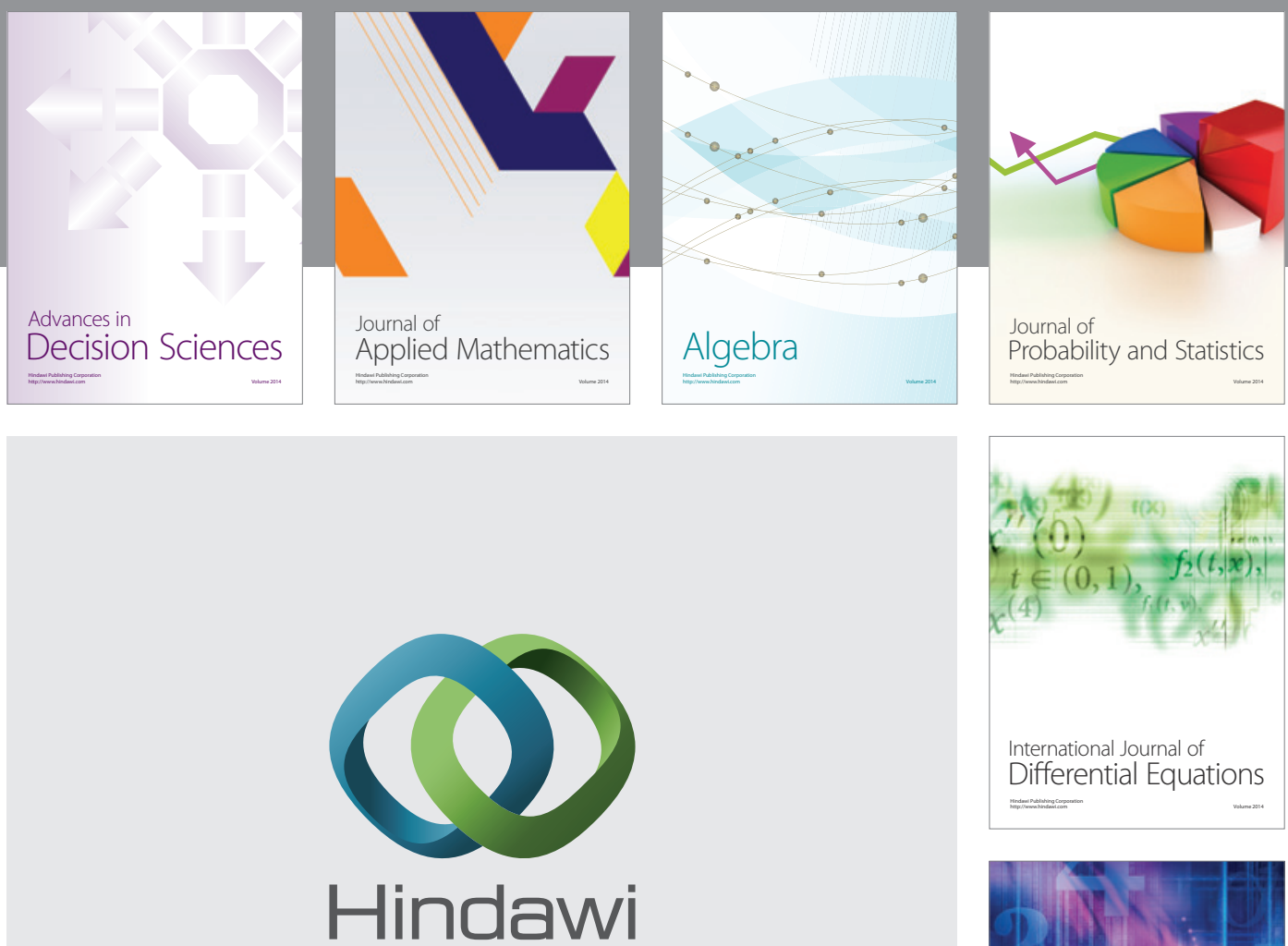

Submit your manuscripts at http://www.hindawi.com
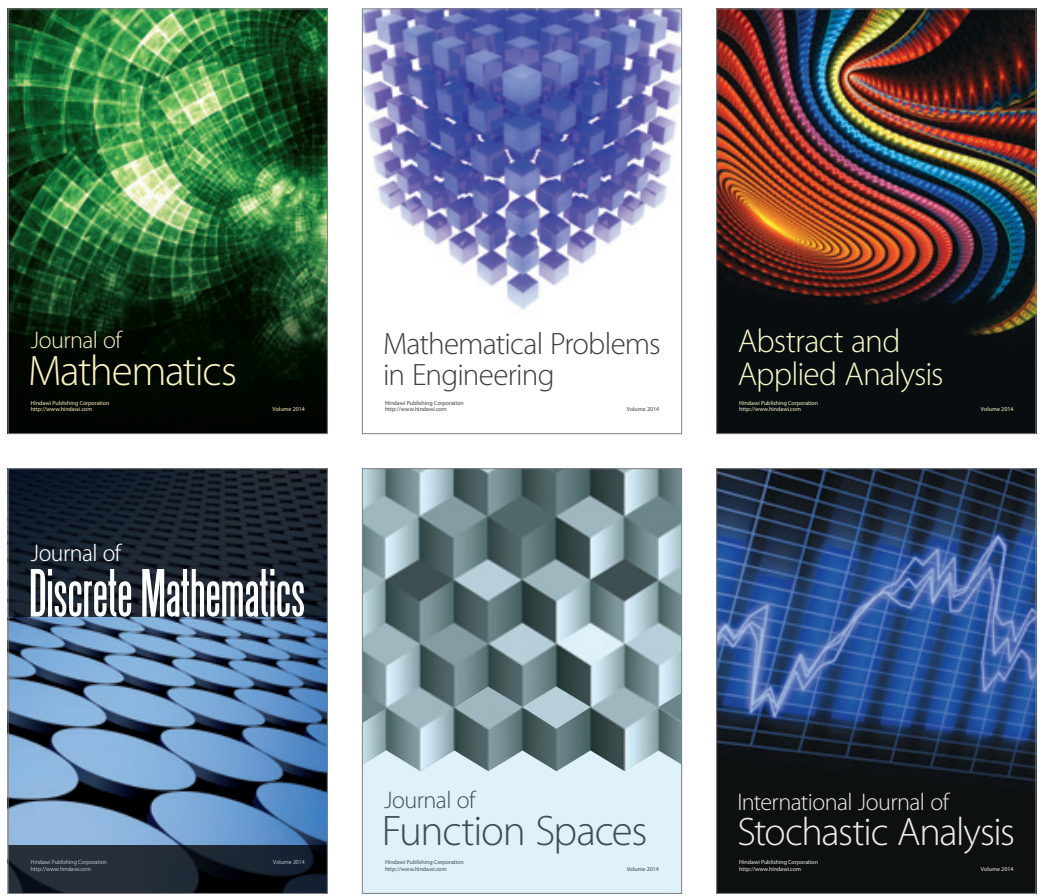

Journal of

Function Spaces

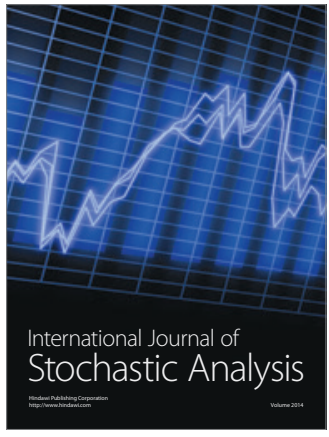

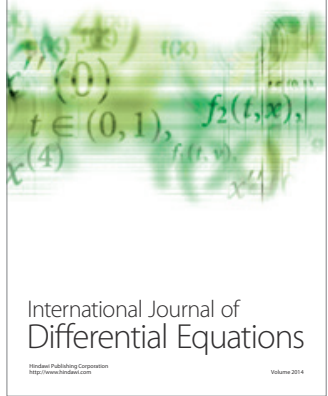
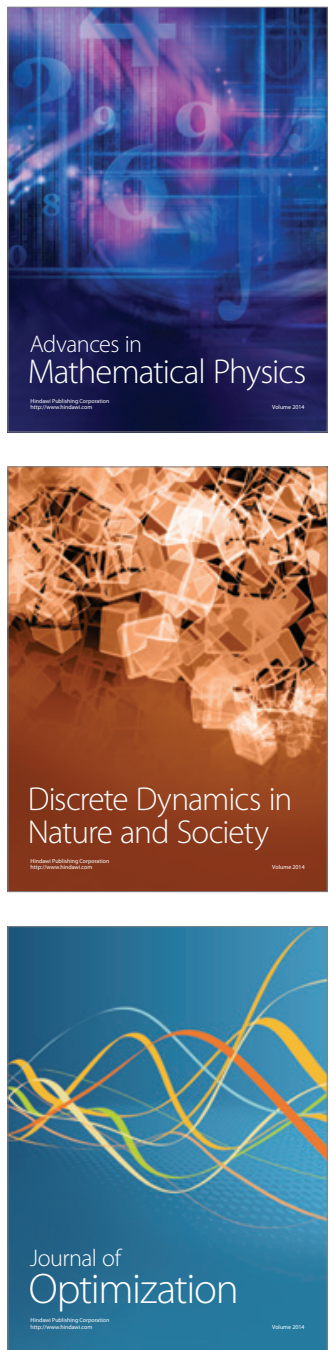